\title{
Cost of off-label antibiotic therapy for bone and joint infections: a 6-year prospective monocentric observational cohort study in a referral centre for management of complex osteo-articular infections
}

\author{
Truong-Thanh Pham ${ }^{1,2,3}$, Eugénie Mabrut ${ }^{2}$, Philippe Cochard ${ }^{4}$, Paul Chardon ${ }^{5}$, Hassan Serrier ${ }^{6,7}$, \\ Florent Valour $^{1,2,8}$, Laure Huot ${ }^{6,7}$, Michel Tod ${ }^{9}$, Gilles Leboucher ${ }^{9}$, Christian Chidiac $^{1,2,8}$, and \\ Tristan Ferry ${ }^{1,2,8}$ \\ ${ }^{1}$ Infectious Diseases Department, Croix-Rousse Hospital, Hospices Civils de Lyon, 69004 Lyon, France \\ ${ }^{2}$ French Referral Centre for complex Bone and Joint Infections, CRIOAc Lyon, 69000 Lyon, France \\ ${ }^{3}$ Division of Infectious Diseases, Department of Medicine, \\ Geneva University Hospitals, 1205 Geneva, Switzerland \\ ${ }^{4}$ Hauteville Public Hospital Centre, 01110 Hauteville-Lompnes, France \\ ${ }^{5}$ Val Rosay Rehabilitation Centre, 69370 Saint-Didier-Au-Mont-d'Or, France \\ ${ }^{6}$ Pôle de Santé publique, Hospices Civils de Lyon, 69003 Lyon, France \\ ${ }^{7}$ Cellule Innovation, Département de la Recherche Clinique et de l'innovation, \\ Hospices Civils de Lyon, 69003 Lyon, France \\ ${ }^{8}$ Centre International de Recherche en Infectiologie (CIRI), Inserm U1111, \\ CNRS UMR5308, ENS de Lyon, UCBL1, 69007 Lyon, France \\ ${ }^{9}$ Service de Pharmacie, Hospices Civils de Lyon, Lyon, France \\ Correspondence: Tristan Ferry (tristan.ferry@univ-lyon1.fr)
}

Received: 5 July 2021 - Revised: 24 August 2021 - Accepted: 25 August 2021 - Published: 7 September 2021

\begin{abstract}
Introduction: Costs related to bone and joint infection (BJI) management are increasing worldwide, particularly due to the growing use of off-label antibiotics that are expensive treatments (ETs), in conjunction with increasing incidence of multi-drug-resistant pathogens. The aim of this study was to evaluate the whole costs related to these treatments during the patient route, including those attributed to the rehabilitation centre (RC) stay in one regional referral centre in France. The total annual cost of ETs for managing complex BJIs in France was then estimated.
\end{abstract}

Material and methods: A prospective monocentric observational study was conducted from 2014 to 2019 in a referral centre for BJI management (CRIOAc - Centre de Référence des Infections OstéoArticulaires complexes). Costs related to expensive treatments ("old" ETs, i.e. ceftaroline, ertapenem, daptomycin, colistin, tigecycline, and linezolid and "new" ETs, defined as those used since 2017, including ceftobiprole, ceftazidimeavibactam, ceftolozane-tazobactam, tedizolid, and dalbavancin) were prospectively recorded. In all cases, the use of these ETs was validated during multidisciplinary meetings.

Results: Of the 3219 patients treated, $1682(52.3 \%)$ received at least one ET, and $21.5 \%$ of patients who received ET were managed in RCs. The overall cost of ETs remained high but stable (EUR 1033610 in 2014; EUR 1129862 in 2019), despite the increase of patients treated by ETs (from 182 in 2014 to 512 in 2019) and in the cumulative days of treatment (9739 to $16191 \mathrm{~d}$ ).

Daptomycin was the most prescribed molecule (46.2\% of patients in 2014 and $56.8 \%$ in 2019, with $53.8 \%$ overall), but its cost has decreased since this molecule was genericized in 2018; the same trend was observed for linezolid. Thus, costs for old ETs decreased overall, from EUR 1033610 in 2014 to EUR 604997 in 2019, but global costs remained stable due to new ET utilization accounting for $46.5 \%$ of overall costs in 2019 . Tedizolid, 
used as suppressive antimicrobial therapy, represented $77.5 \%$ of total new ET costs. In our centre, dalbavancin was never used.

The cost paid by RCs for ETs and the duration of ET remained stable overall between 2016 and 2019.

Conclusions: A high consumption of off-label ET is required to treat patients with BJIs in a CRIOAc, and the consequence is a high cost of antimicrobial therapy for these patients, estimated to be almost EUR 10 million in France annually. Costs associated with ET utilization remained stable over the years. On the one hand, the introduction of the generic drugs of daptomycin and linezolid has significantly decreased the share of old ETs, but, on the other hand, the need for new ETs to treat infections associated with more resistant pathogens has not led to decrease in the overall costs. A drastic price reduction of generic drugs is essential to limit the costs associated with more complex BJIs.

\section{Introduction}

Bone and joint infections (BJIs) are constantly increasing worldwide (Premkumar et al., 2021; Kehrer et al., 2014; Kremers et al., 2015; Rutherford et al., 2016) and have a significant clinical and economic burden. In France, the incidence is estimated at 70 per 100000 persons per year, and in 2013, the total direct cost of treating BJIs was estimated at EUR 421 million (USD 509 million), including EUR 11960 (USD 14 460) per hospital stay (Lemaignen et al., 2021; Laurent et al., 2018; Ferry et al., 2019).

Infections involving an internal device account for up to $57.7 \%$ of complex BJIs (Lemaignen et al., 2021). They are more costly than native BJIs. Indeed, at least one surgery is required, the prevalence of multi-drug-resistant bacteria is higher, and the use of expensive antibiotics is more frequent (Hackett et al., 2015).

Peri-prosthetic joint infections (PJIs) represent the vast majority of these implant infections, and more than onethird of all BJIs (Lemaignen et al., 2021). They are increasing, with a significant morbidity and mortality rate, and high costs. In the USA, projections for 2030 estimate that the annual number of PJIs (hip and knee) could rise to more than 66000 cases per year, with a total cost of more than EUR 1.53 billion (USD 1.85 billion) (Premkumar et al., 2021).

The CRIOAc (Centre de Référence des Infections OstéoArticulaires complexes) network, composed of nine regional referral centres, was implemented in France in 2008 by the General Directorate for the Provision of Healthcare (Direction Générale de l'Offre de Soins; DGOS) for the management of complex BJIs. The complex nature is defined by a patient who presents one or more of the following criteria: (i) relapse, (ii) host-related criteria, e.g. anaesthetic risk terrain, allergy limiting therapeutic management, and history limiting and/or modifying surgical management, (iii) surgical-related criteria, e.g. the need for bone resection or complex bone and/or soft tissue reconstruction, and (iv) pathogen-related criteria, which may be multi-drugresistant with limited therapeutic possibilities (Ministère De La Santé, 2010).
Antibiotic resistance has an impact on the cost of BJIs as it limits the use of orally adequate available drugs and may promote less active or more expensive drug administration. In the USA, the cost related to treatment of BJIs is significantly higher for methicillin-resistant $S$. aureus infections (approximately EUR 88755 or USD 107264 ) than for methicillinsusceptible $S$. aureus infections (approximately EUR 56300 or USD 68 053; Parvizi et al., 2010). The growing incidence of resistant pathogens involved in BJIs, such as multi-drugresistant (MDR) coagulase-negative staphylococci or MDR gram-negative pathogens (Titecat et al., 2013; Da Silva and Salles, 2021), and the occurrence of side effects under conventional treatment are leading to the increasing use of offlabel molecules for the treatment of BJIs.

These antibiotics have sometimes been on the market for more than 10 years and have only been validated for skin and soft tissue infections (anti-gram-positive antibiotics), and urinary tract or intra-abdominal infections (anti-gram-negative antibiotics). The vast majority of them are expensive treatments (ETs). Due to their potential high cost, their use has to be validated during multidisciplinary meetings; therefore, they may be associated with a significant increase in costs of treating BJIs. This could also be a barrier to management in rehabilitation centres (RCs).

There are no precise data in France concerning the volume and cost of prescriptions for ETs. The main objective of this study was to estimate the whole cost of off-label ETs over time for patients treated at CRIOAc in Lyon. We included $\mathrm{RCs}$ and outpatient prescriptions in this descriptive analysis.

\section{Method}

\subsection{Study design}

A prospective monocentric cohort study was conducted at CRIOAc in Lyon (https://www.crioac-lyon.fr, last access: 14 June 2021) and included patients managed for an osteoarticular infection between 1 January 2014 and 31 December 2019. Those patients who refused to participate in the study were excluded. The clinical situation of every patient referred to this referral centre was discussed during multidis- 
ciplinary meetings, and the use of every off-label antibiotic was also validated at these meetings.

\subsection{Study variables and definitions}

Patient and BJI characteristics and prescribing patterns were collected during each visit, including the prescription of antibiotics by RCs or in an outpatient setting. Data were collected on patient's age, sex, body mass index (BMI), American Society of Anesthesiologists (ASA) score, presence or absence of implant, infection type (peri-prosthetic joint infection, osteosynthesis-associated infection, native osteomyelitis, and septic arthritis), time between hardware placement and symptom onset $(<1$ month - acute infection; 1-3 months - subacute infection; $>3$ months - chronic infection), whether the patient was managed by a RC or not, and the start and end dates of antibiotics and their dosage.

ETs were separated into two groups, i.e. "old" and "new", according to their dates of use. Old ETs were ceftaroline, ertapenem, daptomycin, colistin, tigecycline, and linezolid. New ETs, defined as those used from 1 January 2017 onwards, included ceftobiprole, ceftazidimeavibactam, ceftolozane-tazobactam, tedizolid, and dalbavancin. Each ET was prescribed as empirical, targeted, or suppressive therapy.

Peri-prosthetic joint infections (PJIs) and osteosynthesisassociated infections were defined according to the MusculoSkeletal Infection Society (MSIS) 2018 criteria (Parvizi et al., 2018) and the fracture-related infections (FRI) consensus group, respectively (Metsemakers et al., 2018). Osteomyelitis was defined by the Infectious Diseases Society of America (IDSA) definition (Berbari et al., 2015) and septic arthritis by modified Newman's criteria (Mathews et al., 2010).

As RCs were not equipped with the same computerized prescription program, they were asked to fill out a table with the dates of admission and exit of their institution. A crossreference was made between ET dispensation dates and patient care in RCs, making it possible to calculate the exact patient length of stay and duration of treatment (DOT) in RCs.

Eventually, as we know the number of inhabitants that are referred to our centre, the total annual cost attributed to ETs in France was estimated, using the 2018 census for calculation.

\subsection{Statistical analysis}

Categorical variables were described by counts and percentages, while mean and standard deviation or median and interquartile range (IQR) were used to summarize continuous variables. Charts were made with Microsoft Excel for Apple Mac (version 16.48). Description analyses were done with Stata 16.1 (StataCorp LLC, Texas 77845, USA).

\section{Results}

\subsection{Patient characteristics}

Between 1 January 2014 and 31 December 2019, 3219 patients were managed at our referral centre with the following distribution: 410 patients in 2014, 473 in 2015, 524 in 2016, 564 in 2017, 581 in 2018, and 652 in 2019. Of these, 1705 received one or more ET. A total of 23 were excluded from the study (eight declined to participate; 15 did not receive information). Thus, 1682 (52.5\%) were included in the study analysis, i.e. 182 patients $(44.4 \%)$ in $2014,214(45.2 \%)$ in 2015, $220(42.0 \%)$ in 2016, $249(44.1 \%)$ in 2017, 305 $(52.5 \%)$ in 2018, and $512(78.5 \%)$ in 2019.

Patients who received ETs were predominantly male $(n=$ $1048 ; 62.3 \%$ ), with a median age of 64.0 years $(50.0-76.0$ IQR) and a median BMI of $25.8 \mathrm{~kg} / \mathrm{m}^{2}$ (22.8-30.3 IQR). The majority of BJIs were with an internal device $(n=975$; $58.0 \%)$ with a predominance of PJIs $(n=581 ; 60.6 \%$ of implant-associated BJIs) and with an equal proportion of acute $(n=434 ; 25.8 \%)$ and chronic $(n=430 ; 25.6 \%)$ infections. The lower limbs were most frequently affected $(n=$ 1103; 64.5\%; see Table 1).

The part of gram-positive pathogens increased during the study period ( $54.8 \%$ to $65.6 \%)$; it contrasted with decrease in gram-negative BJIs ( $33.2 \%$ to $24.4 \%$ ); over the 6 years, gram-positive bacteria represented more than double the gram-negative bacteria $(n=2534(63.7 \%)$ and $n=1067$ (26.8\%), respectively). Pseudomonas aeruginosa BJIs remained stable over the 6-year study period (approximately $5 \%$; see Table 2).

\subsection{Duration and types of antimicrobial therapy}

Days of treatment with ETs remained stable between 2014 and 2018 but increased by almost 1.5 times in 2019 to reach $16191 \mathrm{~d}$, which is also in line with the increase in patients treated in the referral centre $(n=652$ vs. 410$)$ and the increasing proportion of patients requiring ET $(n=512$ $(78.5 \%)$ vs. $182(44.4 \%))$. The average DOT was reduced by $21.9 \mathrm{~d}$ during the study, i.e. 53.5 in 2014 to $31.6 \mathrm{~d}$ in 2019 . (Table 3; Fig. 1).

Daptomycin was the most commonly prescribed drug, accounting for almost half of treatment days $(n=32503 \mathrm{~d}$; $49.3 \%)$, followed by ertapenem $(n=11914 \mathrm{~d} ; 18.1 \%)$, and linezolid $(n=10409 \mathrm{~d} ; 15.8 \%)$. Use of ertapenem and colistin decreased over the 6-year period at the time of the introduction of ceftazidime-avibactam and ceftolozanetazobactam; utilization of these drugs remained marginal. The remaining ETs were also modestly used, but it is worth mentioning that tedizolid accounted for $14.5 \%$ of ETs in 2019 , mainly due to its use as a suppressive antimicrobial therapy (SAT). Indeed, unlike other centres, dalbavancin was not chosen as potential treatment for BJIs and was particularly not chosen as SAT (Table 4). 
Table 1. Patient and infection characteristics. IQR - interquartile range; BMI - body mass index; ASA - American Society of Anesthesiologists; PJI - peri-prosthetic joint infection; OAI - osteosynthesis-associated infection; ORL - otorhinolaryngology.

\begin{tabular}{ll}
\hline Patient characteristics & $n=1682$ \\
\hline Sex (male), $n(\%)$ & $1048(62.3)$ \\
Age (years), median (IQR; range) & $64.0(50.0-76.0 ; 17.0-91.0)$ \\
BMI (kg/m $\left.{ }^{2}\right)$, median (IQR; range) & $25.8(22.8-30.3 ; 16.2-49.1)$ \\
ASA score, median (IQR) & $2(2-3)$ \\
\hline Infection characteristics & $n=1682$ \\
Presence of internal device, $n(\%)$ & $975(58.0)$ \\
PJI, $n(\%)$ & $568(33.2)$ \\
OAI, $n(\%)$ & $381(22.7)$ \\
PJI+ OAI, $n$ (\%) & $23(1.4)$ \\
Other type of device, $n(\%)$ & $13(0.8)$ \\
Native (without device), $n(\%)$ & $707(42.0)$ \\
\hline Infection location & $n=1709$ \\
Lower limbs, $n(\%)$ & $1,103(64.5)$ \\
Upper limbs, $n(\%)$ & $85(5.0)$ \\
Pelvis, $n(\%)$ & $183(10.7)$ \\
Spine, $n(\%)$ & $121(7.1)$ \\
ORL, $n(\%)$ & $114(6.7)$ \\
Chest, $n(\%)$ & $54(3.2)$ \\
Skull, $n(\%)$ & $37(2.2)$ \\
Other location, $n(\%)$ & $12(0.7)$ \\
\hline Infection chronology $(\%)$ & $n=1682$ \\
Acute $(<1$ month), $n(\%)$ & $434(25.8)$ \\
Sub-acute $(\geq 1$ month and $\leq 3$ months) $n(\%)$ & $111(6.6)$ \\
Chronic $(>3$ months), $n(\%)$ & $430(25.6)$ \\
Not applicable (without device) & $707(42.0)$ \\
\hline Microbiology & $n=3975$ \\
Gram-positive pathogens, $n(\%)$ & $2534(63.7)$ \\
Gram-negative pathogens, $n(\%)$ & $1067(26.8)$ \\
Pseudomonas aeruginosa & $200(5.0)$ \\
Sterile, $n(\%)$ & $374(9.4)$ \\
\hline
\end{tabular}

Table 2. Type of pathogens. BJIs - bone and joint infections.

\begin{tabular}{lrrrrrrr}
\hline & 2014 & 2015 & 2016 & 2017 & 2018 & 2019 & Total \\
\hline Gram-positive pathogens, $n(\%)$ & $241(54.8)$ & $298(60.6)$ & $324(63.2)$ & $381(65.9)$ & $477(67.0)$ & $813(65.6)$ & $2534(63.7)$ \\
Gram-negative pathogens, $n(\%)$ & $146(33.2)$ & $158(32.1)$ & $154(30.0)$ & $148(25.6)$ & $158(22.2)$ & $303(24.4)$ & $1067(26.8)$ \\
Pseudomonas aeruginosa & $20(4.5)$ & $22(4.5)$ & $25(4.9)$ & $39(6.7)$ & $36(5.1)$ & $58(4.7)$ & $200(5.0)$ \\
Culture-negative BJIs, $n(\%)$ & $53(12)$ & $36(7.3)$ & $35(6.8)$ & $49(8.5)$ & $77(10.8)$ & $124(10.0)$ & $374(9.4)$ \\
Total, $n$ & 440 & 492 & 513 & 578 & 712 & 1240 & 3975 \\
\hline
\end{tabular}

\subsection{Expensive treatment costs}

The overall costs related to ETs remained stable between 2014 and 2019 (EUR 1033610 and EUR 1 129 862, respectively), while the number of patients treated, and those for whom ETs were needed, increased. Without new ETs, the costs associated with old ETs would have almost halved between 2014 and 2019 (EUR 1023890 to EUR 604 997), with this being related to the appearance of generic molecules (Tables 3 and 4; Fig. 2).

Daptomycin represented the majority of ET costs $(60.6 \%$ of total costs) but was decreasing since 2016 with the introduction of generic molecules. Indeed, by genericizing the molecule, the price went down from around EUR 150 to EUR 55. In 2019, it corresponded only to $39.2 \%$ of the total costs, whereas in 2016 it was $74.9 \%$, while DOT had doubled (Table 4; Fig. 3). 
Table 3. Number of patients treated in our referral centre and the duration and costs related to expensive treatment. BJIs - bone and joint infections; ET - expensive treatment; RC - rehabilitation centre.

\begin{tabular}{|c|c|c|c|c|c|c|c|}
\hline & 2014 & 2015 & 2016 & 2017 & 2018 & 2019 & Total \\
\hline No. of patients treated for BJIs & 410 & 473 & 524 & 564 & 581 & 652 & 3204 \\
\hline No. of patients treated with ET, $n(\%)$ & $182(44.4)$ & $214(45.2)$ & $220(42.0)$ & $249(44.1)$ & $305(52.5)$ & $512(78.5)$ & $1682(52.5)$ \\
\hline Days of ET & 9739 & 10377 & 8462 & 9525 & 11580 & 16191 & 65874 \\
\hline Mean duration of ET per patient (d) & 53.5 & 48.5 & 38.5 & 38.3 & 38.0 & 31.6 & 39.2 \\
\hline Overall costs (EUR) & 1033610 & 1265520 & 984764 & 1120338 & 1132724 & 1129862 & 6666818 \\
\hline Old ET total costs (EUR) & 1033610 & 1265520 & 984764 & 1005378 & 921843 & 604997 & 5816112 \\
\hline New ET total costs (EUR) & 0 & 0 & 0 & 114960 & 210881 & 524865 & 850706 \\
\hline Percent of new ET costs/overall costs $(\%)$ & 0 & 0 & 0 & 10.3 & 18.6 & 46.5 & 12.8 \\
\hline \multicolumn{8}{|l|}{$\mathrm{RC}$} \\
\hline No. of patients treated in RC, $n(\%)$ & $50(27.5)$ & 49 (22.9) & $22(10.0)$ & $63(25.3)$ & $91(29.8)$ & $87(17.0)$ & $362(21.5)$ \\
\hline Days of ET in RC & $2092(21.5)$ & $1530(14.7)$ & $1473(17.4)$ & $1452(15.2)$ & $1983(17.1)$ & $2406(14.9)$ & $10936(16.6)$ \\
\hline Mean duration of ET per patient in RC (d) & 41.8 & 31.2 & 67.0 & 23.0 & 21.8 & 27.7 & 30.2 \\
\hline Costs of ET in RC (EUR) & 218836 & 173891 & 191098 & 192229 & 200651 & 113848 & 1090553 \\
\hline Percent of ET in RC costs/overall costs (\%) & 21.2 & 13.7 & 19.4 & 17.2 & 17.7 & 10.1 & 16.4 \\
\hline
\end{tabular}
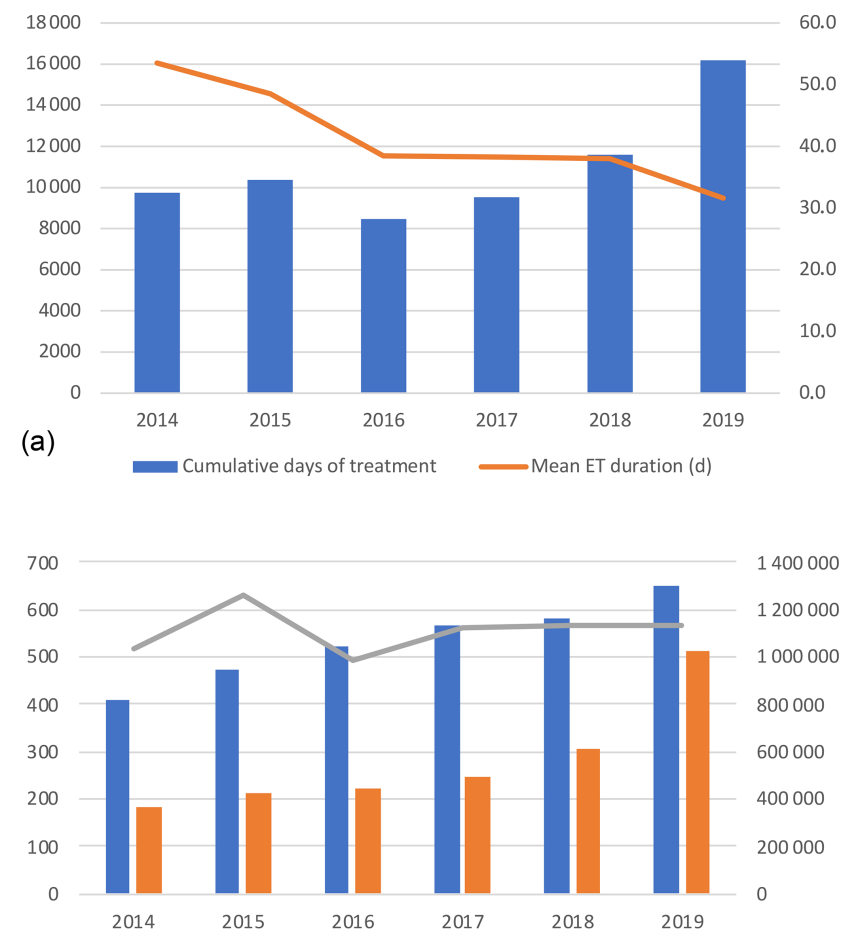

(b)

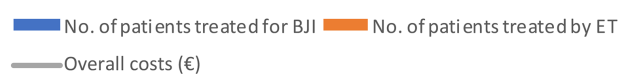

Figure 1. (a) Cumulative days of expensive treatments and mean duration. (b) Number of patients treated (blue) in the referral centre with expensive treatment (orange) and global costs (line). ET expensive treatment; BJI - bone and joint infection.

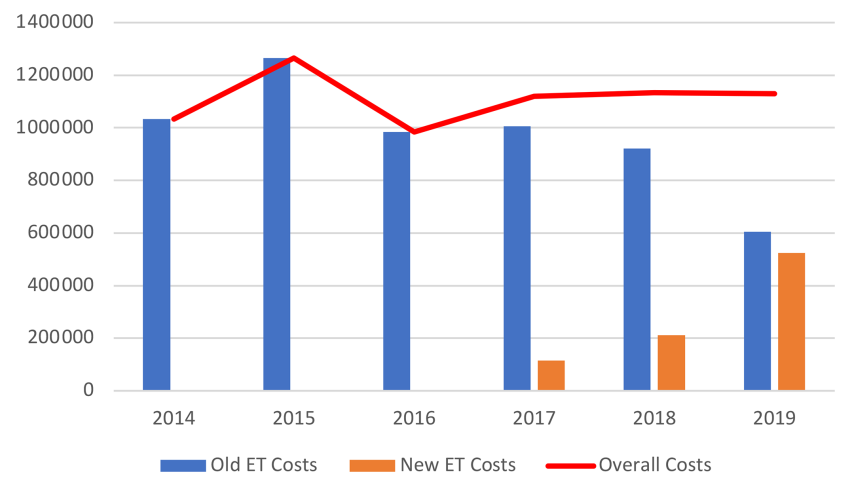

Figure 2. Costs (EUR) related to old and new expensive treatments and total costs. ET - expensive treatment.

The same trend was observed for linezolid with a significant decrease in price, from EUR 122 for 37 patients in $2014(1236 \mathrm{~d})$ to around EUR 5 in 2019 for 137 patients (2904 d). Despite the 2-fold increase in the number of days, total costs related to linezolid decreased by more than 10 times (EUR 150975 to EUR 17818 ).

Ertapenem costs decreased, which is related to the use of new ETs. Indeed, ceftazidime-avibactam and ceftolozanetazobactam have been prescribed since 2017. This use, however, remained marginal compared to treatments targeting gram-positive pathogens.

Eventually, costs related to tedizolid increased drastically in 2019 , representing $36.0 \%$ of the overall costs. This expensive molecule (EUR 175) has been frequently used as a SAT, resulting in long treatment duration and high costs. In 2019, it accounted for the vast majority $(77.5 \%)$ of new ET costs and treatment days $(89.8 \%)$. 
Table 4. Expensive treatments, with the duration and costs by molecule and by year. NA - not applicable; DAP - daptomycin; ETP ertapenem; CST - colistin; TGC - tigecycline; LZD - linezolid; CPT - ceftaroline; TZD - tedizolid; C/T - ceftolozane-tazobactam; CZA ceftazidime-avibactam; BPR - ceftobiprole.

\begin{tabular}{|c|c|c|c|c|c|c|c|c|c|c|c|}
\hline & DAP & ETP & CST & TGC & LZD & $\mathrm{CPT}$ & TZD & $\mathrm{C} / \mathrm{T}$ & CZA & BPR & Total \\
\hline \multicolumn{12}{|l|}{2014} \\
\hline No. of patients & 84 & 37 & 12 & 11 & 37 & 1 & NA & NA & NA & NA & 182 \\
\hline$(\%)$ & $(46.2)$ & $(20.3)$ & (6.6) & $(6.0)$ & $(20.3)$ & $(0.5)$ & NA & NA & NA & NA & \\
\hline No. of days & 4355 & 2981 & 422 & 704 & 1236 & 41 & NA & NA & NA & NA & 9739 \\
\hline$(\%)$ & $(44.7)$ & $(30.6)$ & (4.3) & $(7.2)$ & $(12.7)$ & $(0.4)$ & NA & NA & NA & NA & \\
\hline Costs (EUR) & 609340 & 167051 & 28404 & 70400 & 150975 & 7440 & NA & NA & NA & NA & 1033610 \\
\hline$(\%)$ & $(59.0)$ & (16.2) & $(2.7)$ & $(6.8)$ & (14.6) & $(0.7)$ & NA & NA & NA & NA & \\
\hline \multicolumn{12}{|l|}{2015} \\
\hline No. of patients & 106 & 37 & 11 & 9 & 48 & 3 & NA & NA & NA & NA & 214 \\
\hline$(\%)$ & $(49.5)$ & $(17.3)$ & (5.1) & $(4.2)$ & $(22.4)$ & $(1.4)$ & NA & NA & NA & NA & \\
\hline No. of days & 5267 & 2046 & 756 & 418 & 1551 & 339 & NA & NA & NA & NA & 10377 \\
\hline$(\%)$ & $(50.8)$ & $(19.7)$ & (7.3) & $(4.0)$ & (14.9) & $(3.3)$ & NA & NA & NA & NA & \\
\hline Costs (EUR) & 825198 & 115255 & 46008 & 41300 & 183540 & 54219 & NA & NA & NA & NA & 1265520 \\
\hline$(\%)$ & $(65.2)$ & $(9.1)$ & $(3.6)$ & $(3.3)$ & (14.5) & $(4.3)$ & NA & NA & NA & NA & \\
\hline \multicolumn{12}{|l|}{2016} \\
\hline No. of patients & 129 & 26 & 14 & 13 & 34 & 4 & NA & NA & NA & NA & 220 \\
\hline$(\%)$ & (58.6) & (11.8) & (6.4) & $(5.9)$ & $(15.5)$ & $(1.8)$ & NA & NA & NA & NA & \\
\hline No. of days & 4748 & 1018 & 769 & 714 & 1170 & 43 & NA & NA & NA & NA & 8462 \\
\hline$(\%)$ & $(56.1)$ & $(12.0)$ & $(9.1)$ & (8.4) & (13.8) & $(0.5)$ & NA & NA & NA & NA & \\
\hline Costs (EUR) & 737774 & 64251 & 49029 & 85200 & 42651 & 5859 & NA & NA & NA & NA & 984764 \\
\hline$(\%)$ & $(74.9)$ & $(6.5)$ & $(5.0)$ & $(8.7)$ & $(4.3)$ & $(0.6)$ & NA & NA & NA & NA & \\
\hline \multicolumn{12}{|l|}{2017} \\
\hline No. of patients & 129 & 28 & 16 & 16 & 45 & 3 & 1 & 8 & 2 & 1 & 249 \\
\hline$(\%)$ & (51.8) & $(11.2)$ & (6.4) & $(6.4)$ & $(18.1)$ & (1.2) & $(0.4)$ & (3.2) & $(0.8)$ & $(0.4)$ & \\
\hline No. of days & 4495 & 1885 & 441 & 627 & 1538 & 120 & 3 & 375 & 20 & 21 & 9525 \\
\hline$(\%)$ & $(47.2)$ & (19.8) & $(4.6)$ & $(6.6)$ & $(16.1)$ & (1.3) & $(0.0)$ & (3.9) & $(0.2)$ & $(0.2)$ & \\
\hline Costs (EUR) & 744241 & 96214 & 41575 & 94050 & 6978 & 22320 & 609 & 102765 & 10200 & 1386 & 1118952 \\
\hline$(\%)$ & $(66.5)$ & $(8.6)$ & (3.7) & $(8.4)$ & $(0.6)$ & $(2.0)$ & $(0.1)$ & $(9.2)$ & $(0.9)$ & $(0.1)$ & \\
\hline \multicolumn{12}{|l|}{2018} \\
\hline No. of patients & 166 & 31 & 8 & 15 & 70 & 3 & 5 & 3 & 3 & 1 & 305 \\
\hline$(\%)$ & $(54.4)$ & $(10.2)$ & (2.6) & $(4.9)$ & $(23.0)$ & (1.0) & (1.6) & (1.0) & (1.0) & $(0.3)$ & \\
\hline No. of days & 5627 & 2167 & 220 & 517 & 2010 & 136 & 734 & 125 & 27 & 17 & 9525 \\
\hline (\%) & $(48.6)$ & $(18.7)$ & $(1.9)$ & $(4.5)$ & (17.4) & $(1.2)$ & $(6.3)$ & $(1.1)$ & $(0.2)$ & $(0.1)$ & \\
\hline Costs (EUR) & 681878 & 115847 & 12964 & 77550 & 8308 & 25296 & 124046 & 69750 & 13770 & 3315 & 1129409 \\
\hline$(\%)$ & $(60.4)$ & (10.3) & (1.1) & $(6.9)$ & $(0.7)$ & $(2.2)$ & $(11.0)$ & $(6.2)$ & $(1.2)$ & $(0.3)$ & \\
\hline \multicolumn{12}{|l|}{2019} \\
\hline No. of patients & 291 & 29 & 10 & 7 & 137 & 2 & 24 & 4 & 6 & 2 & 512 \\
\hline$(\%)$ & $(56.8)$ & $(5.7)$ & (2.0) & (1.4) & $(26.8)$ & $(0.4)$ & $(4.7)$ & $(0.8)$ & (1.2) & $(0.4)$ & \\
\hline No. of days & 8011 & 1817 & 497 & 343 & 2904 & 5 & 2347 & 110 & 145 & 12 & 16191 \\
\hline$(\%)$ & $(49.5)$ & $(11.2)$ & (3.1) & $(2.1)$ & (17.9) & $(0.0)$ & (14.5) & $(0.7)$ & $(0.9)$ & $(0.1)$ & \\
\hline Costs (EUR) & 442369 & 86989 & 20976 & 35920 & 17818 & 925 & 406683 & 41990 & 73863 & 2329 & 1127533 \\
\hline$(\%)$ & $(39.2)$ & (7.7) & (1.9) & $(3.2)$ & (1.6) & $(0.1)$ & $(36.1)$ & (3.7) & (6.6) & $(0.2)$ & \\
\hline \multicolumn{12}{|l|}{ Total } \\
\hline No. of patients & 905 & 188 & 71 & 71 & 371 & 16 & 30 & 15 & 11 & 4 & 1682 \\
\hline$(\%)$ & $(53.8)$ & $(11.2)$ & $(4.2)$ & $(4.2)$ & $(22.1)$ & (1.0) & $(1.8)$ & $(0.9)$ & $(0.7)$ & $(0.2)$ & \\
\hline No. of days & 32503 & 11914 & 3105 & 3323 & 10409 & 684 & 3084 & 610 & 192 & 50 & 65874 \\
\hline$(\%)$ & $(49.3)$ & $(18.1)$ & $(4.7)$ & $(5.0)$ & $(15.8)$ & (1.0) & $(4.7)$ & $(0.9)$ & $(0.3)$ & $(0.1)$ & \\
\hline Costs (EUR) & 4040800 & 645607 & 198956 & 404420 & 410270 & 116059 & 531338 & 214505 & 97833 & 7030 & 6659788 \\
\hline$(\%)$ & $(60.7)$ & $(9.7)$ & (8.0) & $(6.1)$ & $(6.2)$ & $(1.7)$ & (3.0) & (3.2) & (1.5) & $(0.1)$ & \\
\hline
\end{tabular}



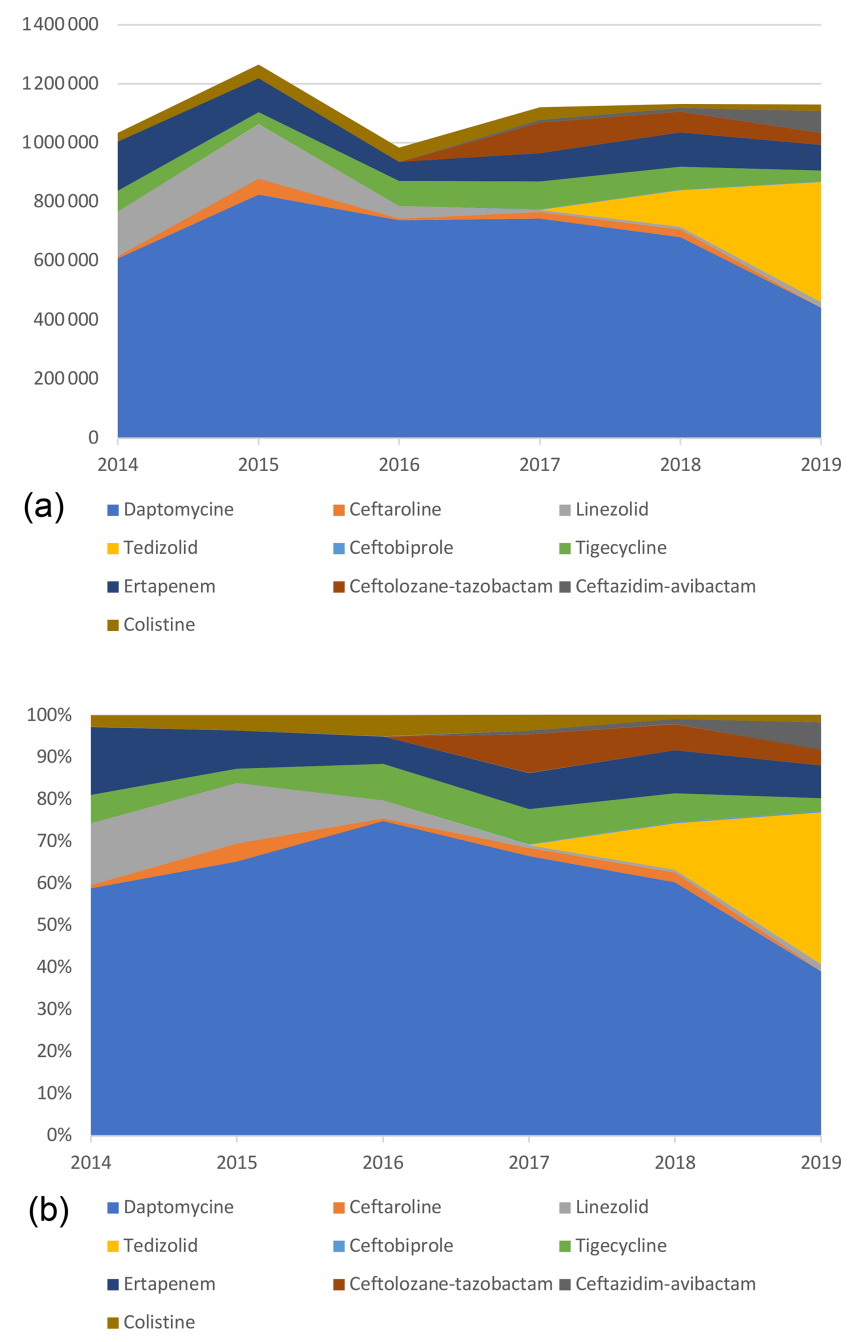

Figure 3. Cumulative (a) and proportional (b) costs (EUR) related to expensive treatments, by molecule.

\subsection{Rehabilitation centre}

Between 2014 and 2019, patients under ETs in RCs increased from 50 in 2014 to 87 in 2019. Nevertheless, the proportion of RC patients receiving ETs compared to the number of patients under ETs remained stable at $21.5 \%$ (Table 3).

The length of stay in RCs decreased between 2014 and 2017 and then increased from $2092 \mathrm{~d}$ in 2014 to $2406 \mathrm{~d}$ in 2019.

The RC global costs related to ETs decreased over this period as follows: EUR 218836 (21.2\% of global ET costs) in 2014, EUR 173391 (13.7\%) in 2015, EUR 191098 (19.4\%) in 2016, EUR $192229(17.2 \%)$ in 2017, EUR 200651 (17.7\%) in 2018, and EUR 113848 (10.1\%) in 2019.

\subsection{Estimation of the global cost in France}

Our referral centre is the dedicated centre for the management of complex BJIs in the Auvergne-Rhône-Alpes region located in the east of France with 7994459 inhabitants. The other part of the country is covered by eight other CRIOAcs. With a total population in France of 68014000 inhabitants, the annual cost due to off-label antibiotics for the treatment of BJIs within the CRIOAc network in France would be almost EUR 10 million euros (EUR 9612 462).

\section{Discussion}

Our study quantified the overall cost of off-label ETs used in BJIs over 6 consecutive years within a CRIOAc. The costs remained globally stable over the study period despite (1) an increasing number of managed patients, (2) a larger portion of these patients requiring ETs, and (3) an increase in cumulative treatment days. This is especially due to the introduction of generic molecules such as daptomycin and linezolid, whose prices, unlike ertapenem, have decreased considerably.

This decrease in costs related to these generic drugs is, however, counterbalanced by the appearance of multi-drugresistant bacteria requiring the use of more expensive, new ETs such as tedizolid. This oxazolidinone antibiotic is similar to linezolid in terms of the spectrum of activity, and it remains active against gram-positive multi-resistant pathogens (Carvalhaes et al., 2019). Unlike linezolid, this antibiotic appears to be better tolerated, with less myelotoxicity and neuropathy and fewer drug-drug interactions (Ferry et al., 2018; Douros et al., 2015). This makes it an excellent choice for SAT, especially for patients with MDR gram-positive PJIs for whom no oral options were available before the market launch of this drug (Ferry et al., 2021). Yet, it remains expensive; in 2019, it accounted for one-third of ET costs and for less than $15 \%$ of cumulative treatment days. It should be noticed that this antibiotic is not available in all countries, such as Switzerland, and that some centres use dalbavancin as a SAT; in our centre, however, it was decided to use tedizolid because it can be taken by mouth, unlike dalbavancin, which requires an injection to be administered in the hospital every 1 or 2 weeks in France (Dinh et al., 2019).

Daptomycin was the most frequently used antibiotic in our study, accounting for almost half of the cumulative days and almost $60 \%$ of the total costs. However, its share of costs has drastically decreased since the introduction of generic drugs in 2018, with a high drop in its price in 2019. It is worth noticing that this ET has been used more since 2018 when the combination of piperacillin-tazobactam and vancomycin was shown to increase the risk of acute renal failure (TriffaultFillit et al., 2018, 2020).

Generic drugs are defined as bio-equivalent replicas of brand name drugs, containing the same active molecules, with identical quality, safety, and efficacy profiles. Only inactive ingredients, like colouring, flavouring, and stabilizing agents can differ (Wouters et al., 2017). Generic drugs can be approved for sale when relevant patents and legal exclu- 
sivities have expired (generally 20 years) or when the patent owner waives their rights (Gulsen Oner and Polli, 2018). Yet, generic drugs have been associated with notable monetary savings. For instance, about $90 \%$ of all prescriptions were filled using a generic drug product in 2019 in the USA, and USD 313 billion could be saved (Association for Accessible Medicines, 2021). Unfortunately, these results do not represent what is has been achieved in other countries. In Switzerland, the proportion of prescriptions filled with generic drugs is only about $17 \%$ to $23 \%$, while in France it is about $30 \%$ to $40 \%$ - far from Germany and Great Britain, with $80 \%$ to $82 \%$ and $83 \%$ to $85 \%$, respectively (Wouters et al., 2017; Decollogny et al., 2011; Organisation for Economic Co-Operation and Development (OECD), 2019). Nevertheless, without the generic molecules of daptomycin and linezolid, the costs associated with ETs during the study period could not have been stable and would have increased dramatically, especially in 2019 with the use of tedizolid, ceftolozane-tazobactam, and ceftazidime-avibactam.

With regard to RCs, their part of the cost was not the majority. The proportion of patients under ETs, the cost paid by RCs for ETs, and the number of cumulative days remained globally stable over the study period.

This study has some other limitations. Indeed, the choice of which antibiotic was an ET was arbitrary and could, therefore, change from one country to another. Moreover, it was a monocentric analysis; the prescription of any antibiotic is, therefore, linked to the practices of the centre and is, therefore, not totally generalizable. Nevertheless, as the patients were treated in a referral centre, it can be assumed that these practices are not so far from the other CRIOAcs in France. Moreover, CRIOAcs are located in hospitals that are mostly members of the same central purchasing group; therefore, they benefit from the same prices negotiated by the laboratories marketing the antibiotics.

Finally, the costs related to antibiotics reflect only part of the costs generated by BJIs. However, in France, the costs are covered by the insurance companies on a fixed-price basis according to the diagnosis code of the disease. Therefore, excess costs related to ETs are supported by the hospitals and also by the healthcare system.

In conclusion, off-label ET use is common for treating BJIs in a referral centre in France, with a huge total cost (estimated to EUR 10 million per year in France), given the entire course of the patient from hospital to RCs and/or outpatient settings. With the introduction of generic molecules of daptomycin and linezolid, overall costs remained stable over the years in our study. Thus, with the rising number of multi-drug-resistant infections, the production of generic antibiotics at low cost is essential to limit the financial burden of the management of BJIs.

Ethical statement. This study was subject to declaration with the local commission for data protection and liberties under number
17_203 and was registered internationally on ClinicalTrial.gov under identifier NCT03335592.

Data availability. The data that support the findings of this study are available from the corresponding author upon reasonable request.

Author contributions. TF participated in the patient care and initiated and designed the study. $\mathrm{PhC}$ and $\mathrm{PaC}$ participated in the patient care and in the collection of the data in RCs. EM, HS, LH, and GL also participated in the collection of the data. TTP checked all the data with EM, performed the descriptive analysis, and drafted the paper. All authors read, revised, and approved the paper.

Competing interests. The contact author has declared that neither they nor their co-authors have any competing interests.

Disclaimer. Publisher's note: Copernicus Publications remains neutral with regard to jurisdictional claims in published maps and institutional affiliations.

Acknowledgements. Lyon Bone and Joint Infection Study Group:

Coordinator: Tristan Ferry. Infectious Diseases specialists: Tristan Ferry, Florent Valour, Thomas Perpoint, Florence Ader, Sandrine Roux, Agathe Becker, Claire Triffault-Fillit, Anne Conrad, Cécile Pouderoux, Pierre Chauvelot, Paul Chabert, Johanna Lippman, and Evelyne Braun. Surgeons: Sébastien Lustig, Elvire Servien, Cécile Batailler, Stanislas Gunst, Axel Schmidt, Eliott Sappey-Marinier, Quentin Ode, Michel-Henry Fessy, Anthony Viste, Jean-Luc Besse, Philippe Chaudier, Lucie Louboutin, Adrien Van Haecke, Marcelle Mercier, Vincent Belgaid, Aram Gazarian, Arnaud Walch, Antoine Bertani, Frédéric Rongieras, Sébastien Martres, Franck Trouillet, Cédric Barrey, Ali Mojallal, Sophie Brosset, Camille Hanriat, Hélène Person, Philippe Céruse, Carine Fuchsmann, and Arnaud Gleizal. Anesthesiologists: Frédéric Aubrun, Mikhail Dziadzko, Caroline Macabéo, and Dana Patrascu. Microbiologists: Frederic Laurent, Laetitia Beraud, Tiphaine RousselGaillard, Céline Dupieux, Camille Kolenda, and Jérôme Josse. Imaging: Fabien Craighero, Loic Boussel, Jean-Baptiste Pialat, and Isabelle Morelec. PK/PD specialists: Michel Tod, Marie-Claude Gagnieu, and Sylvain Goutelle. Clinical research assistant and database manager: Eugénie Mabrut.

Review statement. This paper was edited by Parham Sendi and reviewed by two anonymous referees.

\section{References}

Association for Accessible Medicines: 2020 Generic Drug \& Biosimilars Access \& Savings in the U.S. Report, available 
at: https://accessiblemeds.org/2020-Access-Savings-Report, last access: 14 June 2021.

Berbari, E. F., Kanj, S. S., Kowalski, T. J., Darouiche, R. O., Widmer, A. F., Schmitt, S. K., Hendershot, E. F., Holtom, P. D., Huddleston 3rd, P. M., Petermann, G. W., Osmon, D. R., and Infectious Diseases Society of, A.: 2015 Infectious Diseases Society of America (IDSA) Clinical Practice Guidelines for the Diagnosis and Treatment of Native Vertebral Osteomyelitis in Adults, Clin. Infect. Dis., 61, e26-46, https://doi.org/10.1093/cid/civ482, 2015.

Carvalhaes, C. G., Sader, H. S., Flamm, R. K., and Mendes, R. E.: Tedizolid in vitro activity against Gram-positive clinical isolates causing bone and joint infections in hospitals in the USA and Europe (2014-17), J. Antimicrob. Chemother., 74, 1928-1933, https://doi.org/10.1093/jac/dkz120, 2019.

da Silva, R. B. and Salles, M. J.: Outcomes and Risk Factors in Prosthetic Joint Infections by multidrug-resistant Gram-negative Bacteria: A Retrospective Cohort Study, Antibiotics (Basel), 23, 340, https://doi.org/10.3390/antibiotics10030340, 2021.

Decollogny, A., Eggli, Y., Halfon, P., and Lufkin, T. M.: Determinants of generic drug substitution in Switzerland, BMC Health Serv. Res., 11, 17, https://doi.org/10.1186/1472-6963-1117, 2011.

Dinh, A., Duran, C., Pavese, P., Khatchatourian, L., Monnin, B., Bleibtreu, A., Denis, E., Etienne, C., Rouanes, N., Mahieu, R., Bouchand, F., Davido, B., Lotte, R., Cabaret, P., Camou, F., Chavanet, P., Assi, A., Limonta, S., Lechiche, C., Riou, R., Courjon, J., Illes, G., Lacassin-Beller, F., Senneville, E., and Dalbavancin French Study, G.: French national cohort of first use of dalbavancin: A high proportion of off-label use, Int. J. Antimicrob. Agents, 54, 668-672, https://doi.org/10.1016/j.ijantimicag.2019.08.006, 2019.

Douros, A., Grabowski, K., and Stahlmann, R.: Drug-drug interactions and safety of linezolid, tedizolid, and other oxazolidinones, Expert. Opin. Drug. Metab. Toxicol., 11, 1849-1859, https://doi.org/10.1517/17425255.2015.1098617, 2015.

Ferry, T., Batailler, C., Conrad, A., Triffault-Fillit, C., Laurent, F., Valour, F., Chidiac, C., and Lyon, B. J. I. S. G.: Correction of Linezolid-Induced Myelotoxicity After Switch to Tedizolid in a Patient Requiring Suppressive Antimicrobial Therapy for Multidrug-Resistant Staphylococcus epidermidis Prosthetic-Joint Infection, Open Forum Infect. Dis., 5, ofy246, https://doi.org/10.1093/ofid/ofy246, 2018.

Ferry, T., Seng, P., Mainard, D., Jenny, J. Y., Laurent, F., Senneville, E., Grare, M., Jolivet-Gougeon, A., Bernard, L., Marmor, S., and network, C. R.: The CRIOAc healthcare network in France: A nationwide Health Ministry program to improve the management of bone and joint infection, Orthop. Traumatol. Surg. Res., 105, 185-190, https://doi.org/10.1016/j.otsr.2018.09.016, 2019.

Ferry, T., Conrad, A., Senneville, E., Roux, S., Dupieux-Chabert, C., Dinh, A., Lustig, S., Goutelle, S., Briot, T., Pham, T.-T., and Valour, F.: Safety of tedizolid as suppressive antimicrobial therapy for patients with complex implant-associated bone and joint infection due to multidrug-resistant Gram-positive pathogens: results from the TediSAT cohort study, Open Forum Infect. Dis., 8, ofab351, https://doi.org/10.1093/ofid/ofab351, 2021.

Gulsen Oner, Z. and Polli, J. E.: Authorized Generic Drugs: an Overview, AAPS PharmSciTech, 19, 2450-2458, https://doi.org/10.1208/s12249-018-1073-5, 2018.
Hackett, D. J., Rothenberg, A. C., Chen, A. F., Gutowski, C., Jaekel, D., Tomek, I. M., Parsley, B. S., Ducheyne, P., and Manner, P. A.: The economic significance of orthopaedic infections, J. Am. Acad. Orthop. Surg., 23, S1-7, https://doi.org/10.5435/JAAOSD-14-00394, 2015.

Kehrer, M., Pedersen, C., Jensen, T. G., and Lassen, A. T.: Increasing incidence of pyogenic spondylodiscitis: a 14-year population-based study, J. Infect., 68, 313-320, https://doi.org/10.1016/j.jinf.2013.11.011, 2014.

Kremers, H. M., Nwojo, M. E., Ransom, J. E., Wood-Wentz, C. M., Melton 3rd, L. J., and Huddleston 3rd, P. M.: Trends in the epidemiology of osteomyelitis: a population-based study, 1969 to 2009, J. Bone Joint Surg. Am., 97, 837-845, https://doi.org/10.2106/JBJS.N.01350, 2015.

Laurent, E., Gras, G., Druon, J., Rosset, P., Baron, S., Le-Louarn, A., Rusch, E., Bernard, L., and Grammatico-Guillon, L.: Key features of bone and joint infections following the implementation of reference centers in France, Med. Mal. Infect., 48, 256262, https://doi.org/10.1016/j.medmal.2018.02.004, 2018.

Lemaignen, A., Bernard, L., Marmor, S., Ferry, T., GrammaticoGuillon, L., Astagneau, P., Scientific Committee for Complex, B., and Joint Infections Reference Centers, O. B. O. T. C. N.: Epidemiology of complex bone and joint infections in France using a national registry: The CRIOAc network, J. Infect., 82, 199-206, https://doi.org/10.1016/j.jinf.2020.12.010, 2021.

Mathews, C. J., Weston, V. C., Jones, A., Field, M., and Coakley, G.: Bacterial septic arthritis in adults, Lancet, London, UK, 375, 846-855, 2010.

Metsemakers, W. J., Morgenstern, M., McNally, M. A., Moriarty, T. F., McFadyen, I., Scarborough, M., Athanasou, N. A., Ochsner, P. E., Kuehl, R., Raschke, M., Borens, O., Xie, Z., Velkes, S., Hungerer, S., Kates, S. L., Zalavras, C., Giannoudis, P. V., Richards, R. G., and Verhofstad, M. H. J.: Fracture-related infection: A consensus on definition from an international expert group, Injury, 49, 505-510, https://doi.org/10.1016/j.injury.2017.08.040, 2018.

Ministère de la Santé: INSTRUCTION No. DGOS/PF2/2010/466 du 27 décembre 2010 relative au dispositif de prise en charge des infections ostéo-articulaires complexes 2010, available at: http:// circulaire.legifrance.gouv.fr/pdf/2011/01/cir_32322.pdf (last access: 14 June 2021), 2010.

Organisation for Economic Co-operation and Development (OECD): Health at a Glance 2019: OECD Indicators, available at: https://www.oecd.org/health/health-systems/ health-at-a-glance-19991312.htm (last access: 14 June 2021), 2019.

Parvizi, J., Pawasarat, I. M., Azzam, K. A., Joshi, A., Hansen, E. N., and Bozic, K. J.: Periprosthetic joint infection: the economic impact of methicillin-resistant infections, J. Arthroplasty, 25, 103107, https://doi.org/10.1016/j.arth.2010.04.011, 2010.

Parvizi, J., Tan, T. L., Goswami, K., Higuera, C., Della Valle, C., Chen, A. F., and Shohat, N.: The 2018 Definition of Periprosthetic Hip and Knee Infection: An EvidenceBased and Validated Criteria, J. Arthroplasty, 33, 1309-1314, https://doi.org/10.1016/j.arth.2018.02.078, 2018.

Premkumar, A., Kolin, D. A., Farley, K. X., Wilson, J. M., McLawhorn, A. S., Cross, M. B., and Sculco, P. K.: Projected Economic Burden of Periprosthetic Joint Infection of the Hip 
and Knee in the United States, J. Arthroplasty, 36, 1484-1489, https://doi.org/10.1016/j.arth.2020.12.005, 2021.

Rutherford, A. I., Subesinghe, S., Bharucha, T., Ibrahim, F., Kleymann, A., and Galloway, J. B.: A population study of the reported incidence of native joint septic arthritis in the United Kingdom between 1998 and 2013, Rheumatology (Oxford), 55, 2176-2180, 10.1093/rheumatology/kew323, 2016.

Titecat, M., Senneville, E., Wallet, F., Dezeque, H., Migaud, H., Courcol, R. J., and Loiez, C.: Bacterial epidemiology of osteoarticular infections in a referent center: 10year study, Orthop. Traumatol. Surg. Res., 99, 653-658, https://doi.org/10.1016/j.otsr.2013.02.011, 2013.

Triffault-Fillit, C., Valour, F., Guillo, R., Tod, M., Goutelle, S., Lustig, S., Fessy, M. H., Chidiac, C., Ferry, T., and Lyon, B. J. I. S. G.: Prospective Cohort Study of the Tolerability of Prosthetic Joint Infection Empirical Antimicrobial Therapy, Antimicrob. Agents Chemother., 62, 62, e00163-18, B.J.I.S.G, https://doi.org/10.1128/AAC.00163-18, 2018.
Triffault-Fillit, C., Mabrut, E., Corbin, K., Braun, E., Becker, A., Goutelle, S., Chaudier, P., Fessy, M. H., Dupieux, C., Laurent, F., Gunst, S., Lustig, S., Chidiac, C., Ferry, T., and Valour, F.: Tolerance and microbiological efficacy of cefepime or piperacillin/tazobactam in combination with vancomycin as empirical antimicrobial therapy of prosthetic joint infection: a propensity-matched cohort study, J. Antimicrob. Chemother., 75, 2299-2306, https://doi.org/10.1093/jac/dkaa166, 2020.

Wouters, O. J., Kanavos, P. G., and Mc, K. M.: Comparing Generic Drug Markets in Europe and the United States: Prices, Volumes, and Spending, Milbank Q., 95, 554-601, https://doi.org/10.1111/1468-0009.12279, 2017. 